\title{
Iskra Iveljić
}

\section{Cum ira et studio}

\section{Geschichte und Gesellschaft Kroatiens in den 1990er Jahren"}

\author{
Jedes Jahrhundert hat die Tendenz, sich \\ als das fortgeschrittene zu betrachten, \\ und alle anderen nur nach seiner Idee \\ abzumessen. \\ Leopold von Ranke, Tagebuchblätter, \\ im Anhang von: Weltgeschichte, Text- \\ ausgabe, 4. Bd., 2. unveränderte Auflage, \\ Leipzig 1896, S. 721.
}

\section{Voraussetzungen}

Das Verhältnis zur Geschichte wurde in Kroatien durch drei wichtige allgemeine Faktoren geprägt, nämlich den sozialen, regionalen und ethnisch/nationalen Faktor. Sie führten zur Artikulation äußerst verschiedener Erinnerungskulturen auf dem kroatischen Territorium.

Die unterschiedlichen sozialen Schichten Kroatiens präferierten unterschiedliche Interpretationen der Geschichte. Die oberen Schichten, vor allem der Adel, der lange seine führende soziale Rolle behielt, knüpften an ihre Position als natio politica an die traditionellen Rechte, die sog. iura municipalia ${ }^{1}$, an; sie pflegten den Mythos vom antemurale christianitatis, nach dem das mittelalterliche kroatische Königsreich ein Bollwerk der christlichen Zivilisation gegen die osmanischen Eroberungen gewesen sei. Dieser Mythos basierte auf einem starken Gegensatz (wir, die Christen - sie, die Muslime) und schloß auch Elemente einer Martyrologie ein, die bis heute erhalten blieben: Kroaten haben sich freiwillig und großzügig geopfert, um das christliche Westeuropa vor den Osmanen zu bewahren. Auch für

* Der Text wurde 2002 geschrieben und konnte vor der Veröffentlichung nicht mehr ergänzt werden.

1 Diese Rechte sollten eine gewisse Autonomie, wenn auch keine gleichberechtigte Position gegenüber dem Ungarischen Königreich, mit dem Kroatien seit 1102 in Personalunion verbunden war, gewährleisten. So hatte Kroatien einen eigenen prorex, den Banus, einen eigenen Landtag und dergleichen Dinge mehr. 
die Unterschichten, für das Kleinbürgertum, aber hauptsächlich die Bauern, war dieser Mythos von Bedeutung, da seine einfache Gegenüberstellung (wir - die Feinde) den Bauern half, ihre Identität zu artikulieren. Das Leben auf dem Lande war durch Religion, Bräuche und Legenden geprägt. Die ländlichen Gemeinschaften waren lange physisch, geistig und sozial isoliert, sie lebten in einer eigenen Welt, und sie neigten oft zur Skepsis und Passivität gegenüber der globalen modernen Gesellschaft, die aus den Städten ausstrahlte. Erst seit Anfang des 20. Jahrhunderts wurden sie politisch mobilisiert. Das moderne Bürgertum, insbesondere seine Elite, knüpfte an die alten, ständischen Elemente der Staatlichkeit an und versuchte, sie in eine modernere Form umzuwandeln. Die neue Elite des Bildungs- und Wirtschaftsbürgertums hatte wesentliche Interessen mit der alten Elite gemeinsam, dem Adel und dem hohen Klerus, und arbeitete oft mit ihr zusammen. Ganz anders war die Stellung des Kleinbürgertums, das ein großer sozialer Verlierer im Prozeß der Modernisierung war und deshalb gelegentlich zum Sammelbecken für antisemitische, antiserbische und radikale, großkroatische Optionen wurde. Die Arbeiterbewegung, die Ende des 19. Jahrhunderts entstand, steuerte bis 1918 einen eher gemäßigten Kurs im Sinne des Austromarxismus, und erst nach der Gründung des ersten jugoslawischen Staates entwickelte sie sich in Richtung Kommunismus, mit entsprechender Umstellung des Geschichtsbildes.

Die ethnische/nationale Heterogenität Kroatiens beeinflußte stark die Beziehung zur Geschichte, da verschiedene Ethnien/Nationen auch unterschiedliche Interpretationen der Geschichte pflegten. Besonders zu nennen sind die Differenzen zwischen Kroaten und Serben, aber auch bei den Juden oder den Italienern (in Istrien und Dalmatien) war immer eine andere Erinnerungskultur präsent ${ }^{2}$. Im Unterschied zu den Kroaten konnten die Serben in Kroatien an keine Tradition der Staatlichkeit anknüpfen - nicht einmal an eine virtuelle. Bis zum Zerfall der Habsburgermonarchie konnten sie letztendlich nur eine ziemlich enge, kirchlichschulische Autonomie genießen. In der serbischen Gesellschaft spielte die (Serbische) Orthodoxe Kirche eine große Rolle, und diese Kirche hatte bekanntermaßen eine ganz andere Beziehung zur weltlichen Macht und zur Nation wie die universale römisch-katholische Kirche, die bei den Kroaten dominierte ${ }^{3}$. Deshalb war die Serbische Orthodoxe Kirche ein sehr wichtiger Faktor und Förderer der serbischen Nation. Bei den Kroaten wurde die Beziehung zur Geschichte nicht nur durch den nationalen Faktor geprägt, sondern auch durch die Unmöglichkeit,

2 Bis 1990 waren 12\% der Bewohner Kroatiens Serben. Seit dem kroatisch-serbischen Krieg sind es nur noch 4\%. Die Zahl der Juden wurde durch den Holocaust und die Emigration nach dem Zweiten Weltkrieg drastisch vermindert, und die Volksdeutschen und Italiener wurden nach dem Zweiten Weltkrieg vertrieben. So gab es nach der Volkszählung 1953 nur 413 Juden, 37565 Italiener und 11248 Deutsche in Kroatien.

3 Es gibt bedeutende Wissenschaftler, darunter auch Historiker, die die großen Unterschiede zwischen diesen beiden Kirchen als einen Faktor der Differenzen zwischen Westeuropa und dem Balkan darstellen, vgl. z. B. Holm Sundhaussen, Europa balcanica. Der Balkan als historischer Raum Europas, in: Geschichte und Gesellschaft 25 (1999) 641-644. 
einen kroatischen Staat zu gründen. Als sich endlich im 19. Jahrhundert die moderne kroatische Nation bildete, sie aber ihren Kampf um die eigene volle Staatlichkeit ${ }^{4}$ nicht verwirklichen konnte, wuchs in Kroatien die Geschichte tief in alle Schichten der Gesellschaft ein. Sie durfte nicht nur bloße Vergangenheit sein, sie hatte eine transtemporale Bedeutung, und sie wurde schließlich von verschiedenen Gesellschaftsgruppen zu ihrer Zeitgenossin transformiert. Geschichte besaß keine Autonomie, sie wurde nicht als eine abgegrenzte, beendete Realität verstanden, sondern sie sollte wichtige Aufgaben in der Gegenwart erfüllen. Selbstverständlich unterscheidet sich der Katalog ihrer Aufgaben bei den verschiedenen Gesellschaftsgruppen oder politischen Eliten, aber im Großen und Ganzen bezieht sich ihre Hauptaufgabe auf die Glorifizierung der Nation und die Rechtfertigung der kroatischen Staatlichkeit. Schon die erste Generation der Förderer der modernen nationalen Bewegung in den 1830er Jahren mißbrauchte die Geschichte. Sie aktualisierte die alte (frühneuzeitliche) These der illyrischen Herkunft der Südslawen und versuchte auf diese Weise, Südslawen als alte, autochthone Völker darzustellen, im Gegensatz zu den damaligen kroatischen Hauptfeinden, den Magyaren. Diese erste Phase der nationalen Bewegung ist deshalb als Illyrismus bekannt.

Als den dritten wichtigen Faktor sollte ich die allgemeine Vielfältigkeit Kroatiens erwähnen, die geographisch bedingt ist; aber wichtiger scheint mir die Zugehörigkeit zu verschiedenen historischen Regionen und großen Kulturzonen. Man muß nur auf die jahrhundertlange Zugehörigkeit zur Habsburgermonarchie, zum Osmanenreich und zur venezianischen Republik verweisen. Zwischen den einzelnen kroatischen Regionen bestehen deshalb bis heute krasse Unterschiede, die in der Vergangenheit noch ausgeprägter waren. Diese Regionalismen waren so stark, $\mathrm{da} ß$ bis zum 20. Jahrhundert selbst die Integration der kroatischen Nation auf dem ganzen heutigen Territorium in Frage stand.

Die einzelnen kroatischen Regionen sind geographisch, sprachlich, wirtschaftlich, demographisch und kulturell sehr verschieden. Ich kann nur auf die Unterschiede zwischen dem mediterranen Dalmatien und dem ostmitteleuropäischen Nordkroatien verweisen. Diese regionalen Differenzen beeinflußten auch die Interpretation der Geschichte. Beispielsweise hatte in Dalmatien und Istrien die italienische Kultur eine deutlich andere Bedeutung als im nordwestlichen Kroatien, da in diesen Regionen eine urbane, entweder italienische oder italienisierte Oligarchie bestand. Auch die Helden und Legenden waren unterschiedlich. Diese regionale Heterogenität Kroatiens und ihre Zugehörigkeit zu verschiedenen großen Kulturzonen: der pannonischen, mediterranen und dinarischen, führte letzt-

4 Banalkroatien, d.h. Kroatien, das unter der Verwaltung des Banus und unter der Kompetenz des Kroatischen Landtags stand, genoß seit dem Kroatisch-Ungarischen Ausgleich 1868 eine Autonomie in der inneren Verwaltung, Kultur, im Unterricht und in der Justiz. Da Banalkroatien somit einen Sonderstatus bekam, der nicht nur in Ungarn, sondern in der ganzen Habsburgermonarchie ohne Parallele blieb, konnte die kroatische Elite diesen Status als eine Autonomie mit Elementen der Staatlichkeit interpretieren. 
endlich zu verschiedenen Artikulationen des Kroatentums und stiftete die kroatische Tradition als Pluralität an sich. Jede Singularisierung der kroatischen Identität und Tradition ist deshalb eine Mißinterpretation der Vergangenheit.

Es ist klar, daß Geschichte im 19. Jahrhundert stark instrumentalisiert und den gegenwärtigen Absichten ihrer Interpreten angepaßt wurde, das war überall damals der Fall und Kroatien keine Ausnahme. Die Hauptaufgabe der Geschichte wurde mit der Nation und in der kroatischen Gesellschaft auch mit der Staatlichkeit gekoppelt.

\section{Nach dem Ersten Weltkrieg}

Da der erste jugoslawische Staat, der 1918 entstand, zu keiner echten jugoslawischen Föderation wurde, sondern durch serbische Hegemonie geprägt war, gerieten Serben und Kroaten in heftige Auseinandersetzungen, die nicht allein auf den politischen Bereich begrenzt blieben, sondern Wirtschaft und Kultur umfaßten und selbstverständlich auch die Geschichte. Außer einer relativ dünnen Schicht der politischen und wirtschaftlichen Elite lebten die Völker des ersten Jugoslawien in fast getrennten Welten. Die Serben betrachteten sich als das Piemont und als die Befreier der Jugoslawen, als tapfere Krieger und geschickte Politiker, die den jugoslawischen Staat alleine gründeten ohne Anlehnung an irgendwelche historischen Vorbilder. Sie versuchten, die serbischen Helden, Legenden und Mythen, wie den Kosovo-Mythos ${ }^{5}$, als Staatskultus aufzudrängen, was trotz einiger Sympathien (meist bei den jugoslawisch orientierten Intellektuellen) in Kroatien nie völlig gelingen konnte ${ }^{6}$.

Während des Zweiten Weltkrieges entstand der kroatische Staat (Nezavisna Država Hrvatska - Unabhängiger Staat Kroatien), der als nazistischer Satellit die Rassengesetze proklamierte und KZ-Lager gründete, in denen mehrere Zehntausend Juden, Serben, Roma und Kroaten liquidiert wurden. Der kurzlebige Unabhängige Staat Kroatien artikulierte eine äußerst mißgestaltete, monströse Interpretation der Geschichte zur Rechtfertigung der furchtbaren Verbrechen. So versuchte man zu beweisen, daß Kroaten ihrer Herkunft nach Goten waren. Auf diese Weise sollten die Rassengesetze gerechtfertigt und die Kroaten als „Arier“ dargestellt werden. Man darf nicht vergessen, daß es auch während dieses Regimes

5 Auf dem Kosovo (Amselfeld) fand 1389 eine bedeutende Schlacht gegen die Osmanen statt, in der der serbische Fürst Lazar Hrebeljanović und Sultan Murat I. den Tod fanden. Den Kosovo-Mythos nutzte auch Slobodan Milošević, der gerade dort, am Gazimestan, zum 600. Jahrestag der Schlacht 1989 seine wichtige nationalistische Rede hielt, in der er sein großserbisches Programm klar formulierte.

6 Es ist bemerkenswert, daß bis 1914 in kroatischen Schulbüchern Themen aus der serbischen Geschichte ziemlich gut vertreten waren; entsprechendes galt für Serbien und die kroatische Geschichte nicht. Nach 1918, als diese Themen dann offiziell oktroyiert wurden, gab es Probleme mit der Akzeptanz; siehe Charles Jelavich, Južnoslavenski nacionalizmi (Zagreb 1992). 
möglich war, gute kulturelle Projekte durchzuführen. So wurde gerade in dieser Zeit die Kroatische Enzyklopädie publiziert, die nicht von der exklusiven kroatischen Optik der Ustascha beeinflußt war. Die tragische Zeit des Zweiten Weltkrieges trug zur weiteren Spaltung der Erinnerungskulturen bei. Ein Stereotyp, das bei den Serben weit verbreitet war und dann durch den serbisch-kroatischen Krieg in den 1990er Jahren belebt und verstärkt wurde, ist, daß alle Kroaten der Ustascha angehörten, Faschisten waren, die Serben wiederum die Träger des Antifaschismus waren. Im sozialistischen Jugoslawien wurde deshalb offiziell die Opferzahl der kroatischen KZ Lager absichtlich höher angesetzt, um die antifaschistische, emanzipatorische Orientierung des neuen Regimes zu betonen? ${ }^{7}$. Der Holocaust hat bei den Juden tiefe Spuren hinterlassen. Diese kollektive Angst und das Trauma hatten manchmal eine Vergangenheitsorientierung zur Folge. Die Juden in Kroatien, deren Zahl durch den Holocaust und die Emigration nach dem Krieg sehr gering war, fühlten sich in besonderem Maße als Opfer. Sie bestanden auf dem Antifaschismus, auf dem Standpunkt, daß man die faschistischen Verbrecher jagen müsse und den Holocaust nie vergessen dürfe. Leider muß ich auch feststellen, daß es nach der Gründung des kroatischen Staates 1991 wieder einige antisemitische Töne in der breiten Öffentlichkeit gab, teilweise auch deshalb, weil die Juden wegen ihres Antifaschismus als Förderer des Kommunismus gesehen wurden.

Die Gründung des zweiten Jugoslawien 1945 bedeutete wiederum eine große Wende. Im Unterschied zum ersten Jugoslawien, in dem serbische Hegemonität deutlich war, beruhte das sozialistische Jugoslawien im Prinzip auf der Gleichberechtigung seiner Völker, aber wieder wurde Geschichte für ideologische und politische Zwecke mißbraucht ${ }^{8}$. Einerseits hat man wiederum eine jugoslawische Vertikale in der Geschichte zu sehr betont oder sogar erfunden, andererseits wurde dazu alles, was zur bürgerlichen Gesellschaft gehörte entweder marginalisiert oder auch verboten. Die Relikte des Bürgertums konnten nur in privater Sphäre überleben. Es gab viele Tabuthemen, und bis zum Ende der 1960er Jahre bestanden keine guten allgemeinen Bedingungen für die Entwicklung der Geschichtsschreibung, obwohl eine vulgärmarxistische Option nie weit verbreitet war.

Ich habe absichtlich mit dieser langen Einführung begonnen, da die kroatische Gegenwart noch heutzutage durch die Geschichte geprägt ist. Sarkastisch würde ich sagen, daß wir in vielen Aspekten nicht im 21., sondern im 19. Jahrhundert

7 Man sprach von 700000 Opfern allein im größten KZ-Lager Jasenovac. Der spätere kroatische Präsident Franjo Tuđman wurde als Direktor des Instituts für Geschichte der Arbeiterbewegung in Zagreb 1967 frühzeitig pensioniert, weil er gegen die offiziell zu hoch festgelegte Opferzahl protestierte. Heutzutage spricht man von 50000-70000 Opfern. Interessanterweise kamen zu diesen neuen Zahlen fast zur selben Zeit ein kroatischer und ein serbischer Forscher: Vladimir Žerjavić, Gubici stanovništva Jugoslavije u drugom svjetskom ratu (Zagreb 1989). Bogoljub Kočović, Žrtve drugog svetskog rata u Jugoslaviji (London 1985). 8 Vgl. z.B. Drago Roksandic, Shifting References: Celebrations of Uprisings in Croatia, 1945-1991, in: East European Politics and Societies 9 (1995) 256-271. 
leben. Geschichte spielte immer eine zu große Rolle in Kroatien ${ }^{9}$, und die Mißinterpretationen der Geschichte schwebten oft zwischen einem jugoslawischen und einem exklusiv kroatischen Pol.

\section{Die Suche nach einem neuen Paradigma in den 1990er Jahren}

Kroatien war bis 1991 eine Republik der Sozialistischen Föderativen Republik Jugoslawien, die wiederum eine Sonderstellung innehatte und nie zum Warschauer Pakt und zum „Ostblock“ gehörte. Dieser Sonderweg Jugoslawiens ermöglichte es insbesondere in den 1970er und 1980er Jahren, daß sich die Geschichtsschreibung mehr und mehr vom politischen Einfluß emanzipierte. Die Historiker durften ins Ausland reisen, hatten Zugang zur neuen Fachliteratur, nahmen an internationalen Tagungen teil usw. Die Folge war, daß in den 1970er Jahren eine relevante Bewegung in der Sozialgeschichtsschreibung zu spüren war und in den 1980er Jahren kulturgeschichtliche und Modernisierungsstudien sowie gender studies dazukamen. In den 1980er Jahren begann man, vorsichtig selbst über bisherige Tabuthemen (wie die Zahl der Opfer in Jasenovac, die Rolle der katholischen und orthodoxen Kirche, die Opfer der Nachkriegszeit) zu diskutieren.

Obwohl es so schien, als ob nach dem Fall der Berliner Mauer und dem Kollaps des Kommunismus in Osteuropa die kroatische Geschichtsschreibung gute Bedingungen zur vollen Emanzipation von der Politik hätte und sie sich ziemlich rasch den Entwicklungstendenzen der Geschichtswissenschaft in westeuropäischen Ländern anpassen könnte, war das leider nicht der Fall. Trotz des großen Interesses für Geschichte, das auch von der damaligen politischen Spitze unterstützt wurde (Gründung neuer Hochschulstudien der Geschichte in Zagreb10, Pula, Rijeka und Osijek, wesentlich mehr Mitarbeiter an Instituten, Finanzierung neuer wissenschaftlicher Projekte, intensivere Verlagstätigkeit und dergleichen mehr ${ }^{11}$ ), gab es in den 1990 er Jahren nicht nur beinahe keine bedeutenden neuen

9 Siehe Mirjana Gross, Wie denkt man kroatische Geschichte? Geschichtsschreibung als Identitätsstiftung, in: Österreichische Osthefte 35 (1993) 73-98.

10 Die neuen „Kroatischen Studien“ (Hrvatski studiji) in Zagreb wurden ursprünglich als eine Alternative zu der "roten“ Philosophischen Fakultät gegründet. Sie bestehen auch heute noch und bieten das Studium der kroatischen Kultur und Sprache, der Philosophie, Geschichte und Psychologie an.

11 Die Museen der (antifaschistischen) Revolution in Zagreb und Split sowie die regionalen Institute für Geschichte der Arbeiterbewegung in Slavonski Brod, Split und Rijeka wurden aufgelöst. Das Zagreber Institut desselben Namens jedoch, das von Tuđman gegründet wurde, entwickelte sich, selbstverständlich unter einem anderem Namen (erst Institut für Zeitgeschichte, dann Kroatisches Historisches Institut) zur Stütze des HDZ-Regimes in der Historiographie. Diese Behauptung bezieht sich auf die Führungskräfte des Instituts und nicht auf die Mehrheit seiner Forscher. Die privilegierte Position des Institutes zeigte sich in der Zahl der Mitarbeiter sowie in einer großen Verlagstätigkeit und starken Finanzierung seiner Projekte. 
Strömungen in der Historiographie, sondern Geschichte wurde stärker mißbraucht, als es in den vorhergegangenen 20 Jahren der Fall war.

Dieser scheinbare Widerspruch ist leicht durch den rigiden nationalistischen Druck erklärbar, der nicht nur aus der breiten Öffentlichkeit kam, sondern auch von der damaligen regierenden Partei HDZ (Hrvatska demokratska zajednicaKroatische demokratische Union) unterstützt wurde. Die soziale Rolle der Historiker wurde groß geschrieben, aber sie wurde strikt auf die Apotheose der kroatischen Nation beschränkt. Die Historiker mit dem damaligen Präsidenten Franjo Tuđman an der Spitze sollten beinahe zu Demiurgen der kroatischen Nation und des Staates werden. (Tuđman war kein Berufshistoriker, sondern ein General der Jugoslawischen Armee, aber er interessierte sich stark für Geschichte, gründete 1961 das Institut für Geschichte der Arbeiterbewegung in Zagreb und erlangte den Doktorgrad der Geschichtswissenschaften in Zadar.)

In den 1990er Jahren stand in Kroatien deutlich die Nation im Mittelpunkt, und alles Kroatische in der Geschichte sollte betont oder „erfunden“ werden (um eine luzide Hobsbawm-Phrase zu zitieren $)^{12}$. Man war bemüht, die kroatische Identität von der symbolischen Ebene (neue Fahne und Wappen) bis zur Gründung des kroatischen Heeres endgültig zu stiften. Man benannte Straßen und Institutionen $u^{13}$, feierte neue Feiertage, demolierte viele alte und installierte neue Denkmäler und war überall bemüht, die kroatische Symbolik offiziell festzulegen. Ein gutes Beispiel dafür ist die Reinstallation des Jelačić-Denkmals am Zagreber Hauptplatz. Es wurde 1866 errichtet, und schon zu dieser Zeit war der Banus ein wichtiges Symbol des Kroatentums. Das kommunistische Regime hat das Denkmal deshalb 1947 über Nacht demontieren lassen und seine Zerstörung bestimmt; nur durch Zufall hat es der damalige Direktor der Glyptothek gerettet. Die feierliche Reinstallation, initiiert von der neugegründeten Liberalen Partei, wurde zur groß aufgezogenen Nationalfeier im Jahre 1990, aber selbst dieses Ereignis wurde „uminterpretiert“. Die HDZ nämlich, als sie bei den ersten freien Wahlen siegte, behauptete, sie und nicht die Liberale Partei habe die Reinstallation initiiert.

In dieser Nationaleuphorie schien die Gründung des kroatischen Staates 1991 wichtiger als seine Gestaltung zu einem modernen Kroatien. Es ist zu betonen, daß eine Homogenisierung kroatischer Tradition und Identität stattfand. Man war mehr bemüht, die starken regionalen Differenzen zu marginalisieren und eine einheitliche kroatische Identität zu stiften, statt anzuerkennen, daß es sich eher um verschiedene kroatische Identitäten handelte. So sprach man damals im amtlichen Diskurs nicht über Dalmatien oder Slawonien, sondern über Süd- und Ostkroa-

12 Eric Hobsbawm, Terence Ranger (Hrsg.), The Invention of Tradition (Cambridge 1983).

13 Das Adjektiv „jugoslawisch“" wurde durch die Adjektive „kroatisch“ oder manchmal "südosteuropäisch“ ersetzt, der Genitiv „Kroatiens“ durch „kroatisch“. So wurde z. B. die Jugoslawische Akademie der Wissenschaften und Künste zur Kroatischen Akademie, das Jugoslawische Lexikographische Institut zum Kroatischen Lexikographischen Institut, das Historisches Museum Kroatiens zum Kroatischen Historischen Museum. An der Philosophischen Fakultät änderte man die Namen der Fächer „Geschichte der jugoslawischen Völker" und "Geschichte Jugoslawiens“ in "Geschichte Mittel- und Südosteuropas“. 
tien. Die Verschiedenheit Istriens wurde totgeschwiegen und jede dissonante Stimme aus dieser Region sogleich als Sezession denunziert. Als founding fathers hat man eher konservative oder ausgesprochen national-kroatisch orientierte Personen gewählt, zum eigentlichen pater patriae wurde Ante Starčević ernannt, da sich seine Partei als erste im 19. Jahrhundert für einen selbstständigen kroatischen Staat eingesetzt hatte. Die Rangordnung im Pantheon der nationalen Helden kann man gut an Darstellungen auf den neuen Geldscheinen erkennen. Die größte Nomination (1000 Kuna) gehört Starčević und die kleinste (5 Kuna) den Magnaten Petar Zrinski und Fran Krsto Frankopan, die wegen der Verschwörung gegen die Habsburgermonarchie in Wien 1671 hingerichtet wurden ${ }^{14}$.

Selbstverständlich wurde alles Jugoslawische in der kroatischen Geschichte entweder dämonisiert oder minimiert. So verschiedene Persönlichkeiten wie Ljudevit Gaj (Anführer des Illyrismus), Bischof Josip Juraj Strossmayer (Förderer der jugoslawischen Ideologie seit den 1860er Jahren) und Josip Broz Tito erklärte man beispielsweise zu personae non gratae. Selbst Antifaschismus wurde in Frage gestellt: Die Gründung des nazistischen kroatischen Staates im Zweiten Weltkrieg versuchte man als das Resultat des angeblich gerechten Strebens einer Nation nach eigener Staatlichkeit zu legitimieren. Die Zahl der Opfer der kroatischen KZLager wurde klein geredet, und es bestanden auch Versuche, die ganze UstaschaBewegung zu rehabilitieren. Daß dies nicht völlig gelang, ist der Tatsache zu verdanken, daß Franjo Tuđman ein Partisan und General der Jugoslawischen Armee war, er setzte sich für eine Versöhnung aller kroatischen politischen Richtungen des Zweiten Weltkriegs ein: Partisanen, Ustascha, Landwehr. Tuđman versuchte auch, eine kroatische kommunistische Vertikale zu stiften. Es war eine Tendenz, seine eigene kommunistische Vergangenheit zu rechtfertigen. Deshalb unterstützte er die Initiative, Andrija Hebrang, der als Anhänger der InformbüroResolution arretiert und heimlich liquidiert wurde, als den ersten kroatischen kommunistischen Renegaten herauszustellen, was nicht der Wahrheit entsprach, da Hebrang ein sehr harter und dogmatischer Kommunist war ${ }^{15}$.

Geschichte wurde auch zu antiserbischen und antisemitischen Interpretationen mißbraucht. Die Serbische Orthodoxe Kirche stellte man in einem äußerst negativen Licht, als Stütze der aggressiven großserbischen Option, dar. Man versuchte Kroaten ethnisch von den Südslawen (allen voran den Serben) zu unterscheiden. Dabei wurde auf eine alte iranische Theorie der kroatischen Ethnogenese zurückgegriffen, und auch der alte Mythos vom antemurale christianitatis wurde wiederbelebt, mit dem Unterschied, daß die Rolle der Barbaren, statt wie früher den Türken, jetzt den Serben zugeteilt wurde ${ }^{16}$. Leider wurden auch antisemitische Stim-

14 Scherzhaft habe ich zu meinen Studenten gesagt, daß im Falle von Zrinski und Frankopan zwei kroatische Köpfe nur 5 Kuna (ca. 70 Cent) wert sind.

15 Nada Kisic-Kolanovic, Andrija Hebrang. Iluzije i otrežnjenja (Zagreb 1996).

16 So wurde der Verein für die Erforschung der kroatischen Ethnogenese gegründet. Trotz des gelehrten Namens war die Tätigkeit dieses Vereines eher durch nationalistische Orientierung geprägt. 
men wieder laut. In der regimetreuen und der katholischen Presse erschienen einige antisemitische Artikel. Man versuchte, alle Juden als Kommunisten oder Freimaurer, in jedem Fall aber als Feinde Kroatiens darzustellen. Während der Wahlkampagne des Jahres 1990 äußerte Franjo Tuđman sogar, seine Frau sei glücklicherweise keine Serbin oder Jüdin. Nach heftigen Protesten aus dem Westen korrigierte er auch die ausländische Ausgabe seines Buches ${ }^{17}$, indem er einige antisemitische Behauptungen strich. Selbst unter Berufshistorikern gab es manch antisemitische Töne. Sie denunzierten ihre Kollegen jüdischer Abstammung als Kommunisten und Antikroaten, versuchten zu beweisen, daß es in Kroatien keinen Holocaust gegeben habe. Erst neulich wurde mit gleichen Scheinargumenten ein Buch über den Holocaust in Zagreb kritisiert, weil es ihn angeblich in Zagreb nicht gegeben habe ${ }^{18}$. Dazu wurde die Zahl der Bleiburger Opfer ${ }^{19}$ absichtlich vergrößert und Bleiburg zum neuen Mythos erhoben ${ }^{20}$.

Auch bezüglich Bosniens und der Herzegowina offenbarte sich eine starke großkroatische Option. Es ist allgemein bekannt, daß sich Tuđman das bosnischherzegowinische Territorium mit Serbien teilen wollte. Diese politische Option hatte ihre Befürworter auch unter einigen Historikern und Intellektuellen; sie versuchten zu zeigen, daß Bosnien kroatisch war und ist, die Osmanenherrschaft dagegen nur eine tragische Zwischenperiode dargestellt hatte. Eine wichtige Rolle spielten dabei auch die herzegowinischen Kroaten, die sich stark für die Teilung Bosniens einsetzten. Die herzegowinische Lobby in Kroatien war sehr einflußreich, da in den 1990er Jahren viele Kroaten aus der Herzegowina führende Positionen in Politik, Wirtschaft und Militär innehatten. Nach Meinung einiger Autoren wurde der kroatische Ethno-Nationalismus erst durch die Dynamisierung der bisher in ihrer Tradition erstarrten ländlichen Gebiete, insbesondere in der Herzegowina, angeregt ${ }^{21}$.

Auf der anderen Seite stehen die Tendenzen, die römisch-katholische Kirche mit dem Kroatentum zu identifizieren und ihre positive Rolle in der kroatischen Geschichte und Gegenwart übergroß erscheinen zu lassen 22 . Als Hauptpersönlichkeiten wurden eher konservative Mitglieder der Hierarchie hervorgehoben, wie die Bischöfe Juraj Haulik, Josip Stadler und Alojzije Stepinac, im Gegensatz zu den liberaleren Katholiken wie Strossmayer oder den bosnischen Franziskanern, die sich immer für die Einheit Bosniens einsetzten und oft den katholischen

17 Franjo Tudman, Bespuća povijesne zbiljnosti (Zagreb 1989).

18 Ivo Goldstein, Holokaust u Zagrebu (Zagreb 2001). Die Attacken: Jure Krišto, Goldsteini ponovo osuđuju Stepinca, in: Glas koncila 1 (6.1. 2002). Albert Rebic, Strecha podlegao marksizmu, in: Novi list (11. 1. 2002).

19 Nach dem Krieg wurden kroatische Flüchtlinge (in der Mehrzahl Zivilisten) im Bleiburg von Partisanen liquidiert.

20 Siehe z. B. Andelko Mijatović (Hrsg.), Bleiburg 1941-1995 (Zagreb 1995); Mirko Valentic (Hrsg.), Spomenica. Bleiburg 1945-1995 (Zagreb 1995).

21 Nikša Stančić, Hrvatska nacija i nacionalizam u 19. i 20. stoljeću (Zagreb 2002) 50.

22 Jure Krišto, Prešućena povijest. Katolička crkva u hrvatskoj politici 1850-1918 (Zagreb 1994). Ders., Katolička crkva u totalitarizmu 1945-1990 (Zagreb 1997). 
Kirchenoberen widersprachen ${ }^{23}$. Die katholische Geistlichkeit Kroatiens hat sich jüngst politisch stärker engagiert. So hat sich die Bischofskonferenz zu den Anklagen des Haager Gerichts gegen hohe kroatische Offiziere geäußert.

Durch den Krieg in Kroatien, Bosnien und Herzegowina wurde die kroatische Öffentlichkeit zusätzlich radikalisiert, und während des Kriegs verstummte die zivile Bevölkerung. Die ersten kroatisch-serbischen Auseinandersetzungen begannen nach der ersten demokratischen Wahl 1990, bei der die schon erwähnte HDZ Partei ihren großen Sieg feierte. Die Serben Kroatiens wollten nicht auf den Status einer ethnischen Minderheit reduziert werden und strebten nach der staatlichen Vereinigung mit Serbien und Montenegro. Die neue kroatische politische Führung versäumte es, ihnen konstitutionelle Rechte zu gewähren, und die erste kroatische Verfassung definierte die Republik Kroatien in erster Linie als einen nationalen Staat ${ }^{24}$. Die Serben griffen zu den Waffen, statt politisch zu handeln. Im August 1990 (also noch vor der Unabhängigkeit Kroatiens) blockierten sie die Straßen bei Knin ${ }^{25}$; damit begann die sog. balvan revolucija, die Balkenrevolution. Diese Blockade des öffentlichen Verkehrs konnte die kroatische Seite nicht tolerieren, und nach der Intervention der kroatischen Polizei entwickelte sich der damals noch begrenzte Widerstand, durch die Einmischung der Jugoslawischen Volksarmee (Jugoslavenska narodna armija, JNA) kam es zum Krieg. Ein Drittel des Territoriums der Republik Kroatien wurde von serbischen paramilitärischen Truppen, unterstützt von der JNA, besetzt. Dort proklamierten die Serben ihre autonomen Gebiete. Wegen des plötzlichen serbischen Griffs zu den Waffen und der Intervention der mächtigen JNA fühlten sich die Kroaten als Opfer. Das alte Stereotyp vom Bollwerk der Christenheit wurde wiederbelebt; mehr noch, in der neugegründeten, noch schwachen kroatischen Armee sahen sich die jungen kroatischen Männer (zwischen 20 und 30 Jahre alt) mit einem stärkeren Gegner konfrontiert, so daß es nicht erstaunlich ist, daß gerade bei diesen jungen Leuten die nationalistische Propaganda, die auf der Tradition der Ustascha basierte, auf fruchtbaren Boden fiel. So kam es zu einem wahren Boom der rechtsextremistischen Symbolik: kroatisches Wappen aus der Zeit der NDH, der große Buchstabe „U“ für Ustascha, Hakenkreuz, Bilder des Ustascha-Führers Ante Pavelić und einige seiner berüchtigtesten war lords usw. Die gleiche rechtsnationalistische Symbolik, die an die Tradition der Četnici (der Tschetniks, des serbischen Pendants zu Ustascha) anknüpfte, tauchte bei den Serben auf. Nicht alle Symbole waren jedoch rechtsradikaler Natur. Die kroatische Stadt, die im Krieg am mei-

23 Über Bosnien siehe Noel Malcolm, Bosnia: A Short History (London 1994); Ivo Banac, Cijena Bosne (Zagreb 1994).

24 In der Präambel der Verfassung vom 22. Dezember 1990 wurde die Republik Kroatien als Nationalstaat der Kroaten und als Staat der anderen Völker definiert, von denen namentlich Serben, Muslime, Slowenen, Tschechen, Slowaken, Italiener, Magyaren und Juden erwähnt wurden. Merkwürdig ist, daß die tausendjährige kroatische Staatlichkeit betont und dabei eine lange Liste verschiedener Dokumente und Argumente hinzugefügt wurde, beginnend mit dem 7. Jahrhundert!

${ }^{25} \mathrm{Knin}$ war Zentrum des mehrheitlich von Serben besiedelten Gebietes. 
sten gelitten und schließlich erobert wurde, ist Vukovar. Deshalb wurde die neolithische Taube von Vučedol ${ }^{26}$ zum bedeutenden Symbol des kroatischen Widerstandes, schließlich des ganzen Kroatentums. Es ist schon merkwürdig, daß eine neolithische Plastik zum nationalen Symbol erhoben wird ${ }^{27}$.

Im Krieg wurden zahlreiche Denkmäler zerstört, am meisten kroatische. Sehr oft geschah es durch direkten bewaffneten Angriff, manchmal war es aber auch eine spontane Aktion der lokalen Bevölkerung. Die Zerstörung serbischer Denkmäler fand mehr auf letzterem Weg statt. Insgesamt wurden 2423 der unbeweglichen und 2207 der beweglichen Denkmäler zerstört, beschädigt, oder sie gelten als verschollen. Am stärksten waren die Komitate Dubrovnik-Neretva und Osijek-Baranja betroffen. Dabei muß ich betonen, daß die registrierten Kulturdenkmäler oft das eigentliche Ziel der Zerstörung waren, und daß das Zeichen der Haager Konvention (The Hague Convention sign) ihnen überhaupt keinen Schutz bot, bei manchen katholischen Kirchen hatte man es gerade auf dieses Symbol abgesehen. Daß es sich um einen wahren Denkmalkrieg handelte, kann ich auch mit der Tatsache belegen, daß am meisten zivile Denkmäler wie alte Häuser, Schlösser (1759) und Religionsdenkmäler (495) sowie Kirchen und Klöster ${ }^{28}$ beschädigt wurden.

Der Krieg breitete sich auf Bosnien und die Herzegowina aus, und dort wurde er letztendlich zur furchterregenden und unkontrollierbaren Eskalation von Gewalt seitens aller drei Parteien. Es muß jedoch betont werden, daß sowohl in Kroatien wie in Bosnien der Konflikt zuerst von serbischer Seite ausging 29.

Unter diesen Bedingungen konnten die Historiker ihre Arbeit nur mit Mühe sine ira et studio ausführen. Eine kleine Gruppe stellte sich ganz in den Dienst des HDZ-Regimes und hielt sich nicht an die Professionalität der Geschichtsschreibung. Diese Gruppe war bereit, Mythen und Legenden als historische Wahrheit zu präsentieren, insbesondere in den Schulbüchern.

Hier kommen wir zu einem wichtigen Aspekt des Mißbrauchs von Geschichte. Da die Historiker sich relativ erfolgreich dem nationalistischen Druck widersetzten und das HDZ-Regime zu keinen strengen Maßnahmen gegen diese Opponen-

26 Vučedol bei Vukovar ist ein bedeutender Fundort der neolithischen Kultur im Donauraum.

27 Ein anderes Beispiel ist der "Heimataltar" (Oltar domovine), der auf der mittelalterlichen Burg Medvedgrad bei Zagreb errichtet wurde. Es besteht jedoch ein wesentlicher Unterschied zu der Taube von Vučedol, da der Bau von der HDZ und Tudman selbst initiiert wurde.

28 War-Inflicted Damage on Cultural Monuments 1991-1995, Final Report, Republic of Croatia - Ministry of Culture - Agency for the Protection of Cultural Heritage (Zagreb 1999).

29 Ich muß, wenn auch nur in einer Fußnote, hinzufügen, daß man im Zusammenhang mit dem Krieg in Ex-Jugoslawien im Westen manchmal zu Stereotypen griff, um das Geschehen zu erklären. Es war so einfach, diese tragischen Ereignisse, insbesondere den Genozid, als einen inhärenten Teil des Balkansyndroms zu definieren und alle Nationen dieser Region als wilde, unzivilisierte barbarische Stämme darzustellen. Siehe darüber Maria Todorova, Die Erfindung des Balkans (Darmstadt 1999). 
ten griff, durfte die wissenschaftliche Tätigkeit als solche frei ausgeübt werden. Die Schulbücher wurden jedoch strengstens nach nationalistischen Kriterien überprüft. Als Autoren tauchten manchmal anonyme und äußerst inkompetente Historiker auf, die jedoch bereit waren, alles Kroatische in der Geschichte entweder als älter oder besser, als es wirklich war, darzustellen. So schrieb man über ununterbrochene kroatische Staatlichkeit seit dem 7. Jahrhundert ${ }^{30}$ oder über die feierliche Krönung des ersten kroatischen Königs im 10. Jahrhundert auf einer großen Nationalversammlung31. Selbstverständlich gab es diese Krönung nicht. Den Schülern wurden nicht nur gefälschte, kroatozentrische, sondern auch ziemlich xenophobische, antiserbische Texte geboten. Alle Opponenten dieser Option wurden prompt als Kommunisten, "Jugounitaristen" 32 , Marxisten - mit einem Wort als Anti-Kroaten etikettiert ${ }^{33}$. Es stellte sich heraus, daß die Dominanz des nationalistischen Diskurses in den Schulbüchern nicht auf Kroatien begrenzt war. Beinahe derselbe Diskurs, aber serbischer und bosnisch-moslemischer Prägung, fand in den Geschichtsschulbüchern in Bosnien, Herzegowina, Serbien und Montenegro statt. Komparative Analysen lassen die Schlußfolgerung zu, daß die Geschichtsschulbücher ein Medium der Konstruktion aktueller gesellschaftlicher Realität durch eine Meistererzählung über die Vergangenheit sind. Das Ziel ist eindeutig die Bildung eines kämpferischen, epischen und pathetischen Geistes: durch Schaffung von Metaphern, die Gewalt ausdrücken, oder durch eine fokussierte Perspektive - wir (Kroaten, Europäer, Christen), ihr die anderen (Serben, Muslime usw.). Es wurde behauptet, daß Kroaten Kriege ausschließlich zu ihrer Verteidigung führten, daß sie oft Opfer anderer Völker wurden, Beispiele positiver interethnischer Zusammenarbeit blieben nahezu unerwähnt. Es fehlten auch Kapitel über ethnische Minderheiten, Frauen- und Jugendgeschichte, Antifaschismus, die ein wichtiger Bestandteil von Schulbüchern in Westeuropa sind ${ }^{34}$. Fast zu gleichen Ergebnissen kamen die Untersuchungen in Serbien ${ }^{35}$.

Erst nach einem erbitterten und heftigen Kampf, der in aller Öffentlichkeit geführt wurde, konnten sich einige angesehene Historiker und Lehrer als Schul-

30 Dragutin Pavličević, Povijest Hrvatske (Zagreb 22000) 14.

31 Večernji list (Zagreb, 5. 10. 1997).

32 D.h. die Anhänger der unitären jugoslawischen Option.

33 Solche Interpretation konnte man in folgenden Schulbüchern finden: Filip Potrebica, Dragutin Pavličevic, Povijest (Zagreb 1997); Ivo Peric, Povijest 8 (Zagreb 1996); Ivo Makek, Povijest 6 (Zagreb 1997). Vgl. auch die Diskussion in der Presse: Miroslav Brandt, Teror unitarizma i boljševizma, in: Skolske novine (10.12. 1991). Ivan Biondić, in: Skolske novine (6. 6. 2000).

34 Ivan Ivas, Mitologiziranje i ideologiziranje prošlosti, in: Školske novine 15-16 (18. 4. 2000). Vgl. auch Damir Agičic, Slika naroda jugoistočne Europe u hrvatskim udžbenicima povijesti za osnovnu školu, in: Radovi Zavoda za hrvatsku povijest 31 (Zagreb 1998) 205215.

35 Eine Analyse hatte z.B. Dubravka Stojanović von der Philosophischen Fakultät in Belgrad durchgeführt. Vgl. Zarez, (Zagreb 5. 3. 1999). Einen guten Überblick über die komparative Analyse von Schulbüchern in Südosteuropa bietet das Projekt des Georg Eckert Instituts. Website von South-east Europe Textbook Network: www.gei.de/english/projekte/ southeast.shtml. 
buchautoren in Kroatien durchsetzen. Nach der Wahlniederlage der HDZ im Jahr 2000 wurden die Schulbücher neu geschrieben, aber das alte Schulprogramm, das als obligatorischer Rahmen allen Schulbüchern dient, merkwürdigerweise noch nicht geändert.

Es ist offensichtlich, daß Geschichte in den 1990er Jahren zum Opfer verschiedener Mißinterpretationen wurde, da man bemüht war, aus der Geschichte die Legitimation für die Gegenwart, d.h. für die kroatische Staatlichkeit, zu schöpfen. Gerade deshalb konnten sich mehrere national orientierte schlechte Historiker oder gebildete Fachfremde (wie Theologen) als gefeierte Historiker durchsetzen. Manche von ihnen kamen aus der kroatischen Diaspora, ihre Arbeiten und öffentlichen Auftritte wurden in der breiten Öffentlichkeit relativ wohlwollend aufgenommen. Selbst Historiker, die sich für die Professionalität einsetzten, wurden durch die allgemeine eher nationalistische gesellschaftliche Atmosphäre in der Auswahl ihrer Themen und insbesondere in der Fokussierung auf die nationale Geschichte beeinflußt. Obwohl auch die kroatische Ethnogenese und das Mittelalter erforscht wurden, galt das besondere Interesse der neueren kroatischen Geschichte (19.-20. Jahrhundert), als Zeit der Gestaltung einer modernen bürgerlichen Gesellschaft und Nation. Man beschäftigte sich besonders mit der Nachkriegszeit und ihren vielen bisherigen Tabuthemen, z.B. der Vertreibung der Volksdeutschen und Italiener, der Rolle von Erzbischof Alojzije Stepinac und der Katholischen Kirche überhaupt, den Massakern an Zivilisten in Bleiburg, den 1968er Jahren und der 1971 er Bewegung, dem sogenannten kroatischen Frühling. Auch die neuesten Geschehnisse seit dem Fall der Berliner Mauer wurden schon von einigen seriösen Wissenschaftlern bearbeitet ${ }^{36}$. Es dominieren Themen aus der politischen, nationalen und Ideengeschichte, trotzdem gibt es einige Arbeiten zur Sozial-, Kultur-, Wirtschafts- und Alltagsgeschichte sowie gender studies. Es existiert ein ausgesprochener Kroatozentrismus, man findet fast keine Studien zur europäischen oder Weltgeschichte, obwohl der Europadiskurs sehr ausgeprägt ist, sowohl in der Geschichtsschreibung ${ }^{37}$ als auch in der Öffentlichkeit. Während der Europabegriff stark mit positiven Konnotationen beladen ist, bleibt der Terminus „Balkan“, insbesondere in der breiten Öffentlichkeit, ein Sammelbecken für negative Konnotationen wie Primitivismus, Krieg usw. ${ }^{38}$.

Ein weiteres großes Defizit der Historiographie ist der Mangel an neuen Methoden. Als Ausnahmen kann ich die Anwendung der oral bistory auf die Bauerngeschichte in der Zwischenkriegszeit erwähnen und die Versuche, die linguistischen Ansätze (der sogenannten linguistischen Wende) auf Geschichte zu appli-

36 Dušan Bilandžic, Hrvatska moderna povijest (Zagreb 1999). Ivo Goldstein, Croatia. A History (London 1999). Ivo Banac, Raspad Jugoslavije: eseji o nacionalizmu i nacionalnim sukobima (Zagreb 2001).

37 Die Kroatische Akademie der Wissenschaften und Künste gibt eine große Edition über Kroatien und Europa heraus. Hrvatska i Europa: kultura, znanost i umjetnost, bisher 3 Bde. (Zagreb 1997, 2000 und 2004).

38 Dunja Ribtman Auguštin, Zašto i otkad se grozimo Balkana?, in: Erasmus 19 (Zagreb 1997) 27-35. 
zieren $^{39}$. Trotz einiger guter Arbeiten zur Alltagsgeschichte und die gender studies betreffend, muß man feststellen, daß gerade in bezug auf die Bauern-, Familienund Alltagsgeschichte die kroatischen Ethnologen, die die besten Elemente der Kulturanthropologie rezipierten, den Historikern ziemlich weit voraus sind.

Während des Krieges wurde auch die Kommunikation mit Kollegen aus anderen ehemaligen jugoslawischen Republiken (außer Slowenien) abgebrochen; so konnte man z. B. in Zagreb lange keine serbischen Bücher kaufen. Die Kontakte wurden dann in der zweiten Hälfte der 1990er Jahre wieder zaghaft aufgenommen, angeregt durch verschiedene internationale Institutionen und Stiftungen ${ }^{40}$.

Selbst die Kommunikation unter kroatischen Historikern war nicht immer die beste. Es existierte eine Spaltung zwischen eher nationalistisch orientierten Historikern und denjenigen, die sich dieser Tendenz mehr oder weniger widersetzten. So fand erst 1999 der erste kroatische Historikertag statt. Er war durch Kompromisse geprägt, weil man einen offenen Konflikt zwischen den beiden oben genannten Historikergruppen vermeiden wollte. Deshalb war es letztendlich auch nicht möglich, daß Tuđman den Eröffnungsvortrag hielt ${ }^{41}$, und deshalb befaßten sich die Teilnehmer mit den großen Themen der Nationalgeschichte wie der kroatischen nationalen und staatlichen Identität und Kontinuität, aber auch mit dem Bild des Anderen, Frauengeschichte z.B. ${ }^{42}$.

Trotz mehrerer ausgezeichneter Arbeiten übte gerade die Elite der Historiker selbst keinen großen Einfluß auf die eigene Zunft, geschweige denn auf die Öffentlichkeit, aus. Trotz des ungünstigen gesellschaftlichen Klimas haben die besten Historiker in den 1990er Jahren ihre Arbeiten in der Stille ihrer Arbeitszimmer geschrieben. Ihre Rezeption, im Unterschied zu den nationalistisch intonierten Arbeiten, blieb fast aus.

Abschließend würde ich sagen, daß, obwohl man in Kroatien viel über Deideologisierung gesprochen hat, eher der Versuch einer Reideologisierung der Geschichte in den 1990er Jahren stattfand. Die Fehlinterpretation und der Mißbrauch der Geschichte spielten eine wichtige Rolle im Versuch, die kroatische Gegenwart nach den nationalistischen Vorstellungen zu gestalten. Es bleibt zu hoffen, daß diese Tendenz endgültig vorbei ist, und daß sich besonders die jüngste Generation der Historiker wieder ihrer Arbeit sine ira et studio widmen kann.

39 Suzana Leček, Nismo meli vremena za igrati se... Djetinjstvo na selu (1918-1941), in: Radovi 30 (1997) 209-244. Vladimir Biti, Strano tijelo pri/povijesti (Zagreb 2000).

40 Seit 1998 finden beispielsweise regelmäßige Treffen kroatischer und serbischer Historiker statt, die durch die Friedrich Naumann-Stiftung gesponsert und initiiert werden. Vgl. Hans Georg Fleck, Igor Graovac (Hrsg.), Dijalog povjesničara-istoričara, Bd. 4 (Zagreb 2001).

41 Ein wichtiger Grund, warum er diesen Vortrag nicht halten konnte, war seine sehr schwere Krankheit. Der Historikertag wurde dann tatsächlich wegen Tuđman's Tod abgebrochen.

42 I. Kongres hrvatskih povjesničara. Hrvatski nacionalni i državni identitet-kontinuitet. Knjiga sažetaka (Zagreb 1999). S. die Beiträge auch in: Historijski zbornik 59 (Zagreb 1999) 105-192. 